\title{
ICINet: ICI-Aware Neural Network Based Channel Estimation for Rapidly Time-Varying OFDM Systems
}

\author{
Yi Sun, Student Member, IEEE, Hong Shen, Member, IEEE, Zhenguo Du, Lan Peng, \\ and Chunming Zhao, Member, IEEE
}

\begin{abstract}
A novel intercarrier interference (ICI)-aware orthogonal frequency division multiplexing (OFDM) channel estimation network ICINet is presented for rapidly time-varying channels. ICINet consists of two components: a preprocessing deep neural subnetwork (PreDNN) and a cascaded residual learning-based neural subnetwork (CasResNet). By fully taking into account the impact of ICI, the proposed PreDNN first refines the initial channel estimates in a subcarrier-wise fashion. In addition, the CasResNet is designed to further enhance the estimation accuracy. The proposed cascaded network is compatible with any pilot patterns and robust against mismatched system configurations. Simulation results verify the superiority of ICINet over existing networks in terms of better performance and much less complexity.
\end{abstract}

Index Terms-Channel estimation, deep learning, orthogonal frequency division multiplexing (OFDM), rapidly time-varying channel

\section{INTRODUCTION}

Fifth Generation (5G) and beyond 5G (B5G) wireless communication systems are envisioned to support reliable data transmissions even under the challenging high mobility scenario [1]. When the time-domain channel varies rapidly, the performance of the widely used orthogonal frequency division multiplexing (OFDM) modulation can be severely degraded due to the non-negligible intercarrier interference (ICI) caused by the Doppler shift. To address this problem, it is necessary to develop advanced OFDM channel estimation schemes that can adapt to the fast time-varying channel.

Recently, deep learning has drawn widespread attentions in the area of wireless physical-layer techniques [2]-[4]. In particular, the deep learning based OFDM channel estimation has been investigated in a number of prior works such as [5][10]. As a first attempt, [5] introduced a fully-connected neural network to recover the transmitted symbols directly, whereas the channel was estimated in an implicit way. Alternatively, concerning the channel estimation and signal detection for OFDM systems, the authors of [6] adopted a model-driven approach by incorporating the expert knowledge into the neural network design. However, these two works did not consider time-varying channels. In [7], a channel estimation

Y. Sun, H. Shen, and C. Zhao are with the National Mobile Communications Research Laboratory, Southeast University, Nanjing 210096, China (e-mail:\{sun_yi, shhseu, cmzhao\}@seu.edu.cn). C. Zhao is also with Purple Mountain Laboratories, Nanjing 211111, China.

Z. Du and L. Peng are with Huawei Device Co., Ltd, Shenzhen 518129, China (e-mail:\{zhenguo.du, penglan1\}@huawei.com). network called ChanEstNet was developed for high mobility scenarios using block-type pilot patterns. Furthermore, the work was extended to the multiple-input-multiple-output (MIMO) setup in [8]. The similarity between the pilot-based channel estimation and the super resolution (SR) technique in image processing was exploited in [9], [10] to yield a high-quality channel estimate for OFDM systems. Treating the time-frequency response of a doubly selective channel as a two-dimensional (2D) image, the authors of [9] and [10] proposed ChannelNet based on the convolutional neural network and ReEsNet based on the residual network, respectively. It is worthwhile noting that the aforementioned neural network based channel estimation methods do not explicitly take the impact of ICI into account, which can lead to performance degradation especially in presence of rapid channel variations.

In this work, we propose a novel ICI-aware channel estimation network ICINet, which is superior to existing networks in terms of both performance and complexity. Specifically, ICINet consists of a preprocessing deep neural subnetwork (PreDNN) and a cascaded residual learning-based neural subnetwork (CasResNet). The PreDNN, which includes the ICI information on a few adjacent subcarriers into the network input, is used to refine the least square (LS) based initial channel estimates, while CasResNet can further improve the accuracy of the channel estimates over the 2D time-frequency grid. Owing to the model-driven strategy and the parametersharing mechanism, the proposed cascaded network is adaptive to different pilot patterns and system configurations. In addition, as a preprocessing network, the proposed PreDNN can be readily cascaded with other networks for performance enhancement with only a slight increase in the complexity.

\section{SyStem MOdeL}

Consider an OFDM system over doubly selective fading channels, where each subframe is constituted by $K$ subcarriers and $T$ OFDM symbols. In order to suppress the inter-symbol interference (ISI) caused by the multipath effect, a cyclic prefix (CP) with length $N_{C P} \geq N_{L}-1$ is added before each OFDM symbol, where $N_{L}$ denotes the number of channel taps. It was usually assumed that the CIR remains constant within the duration of one OFDM symbol in prior works such as [5], [6], which applies for slowly time-varying channels. However, for the high mobility scenario, this assumption no longer holds [11]. In fact, the fast channel variation destroys the orthogonality among subcarriers and leads to severe ICI. Consequently, 
after removing the $\mathrm{CP}$ and performing the discrete Fourier transform (DFT), the frequency-domain received signal of the $t$-th OFDM symbol $\mathbf{Y}_{t} \in \mathbb{C}^{K \times 1}$ can be expressed as

$$
\mathbf{Y}_{t}=\mathbf{H}^{(t)} \mathbf{X}_{t}+\mathbf{W}_{t}, \quad t=1, \cdots, T,
$$

where $\mathbf{X}_{t} \in \mathbb{C}^{K \times 1}$ and $\mathbf{W}_{t} \in \mathbb{C}^{K \times 1}$ are the frequencydomain transmitted symbols and zero-mean additive white Gaussian noise with covariance $\sigma^{2} \mathbf{I}$, respectively. $\mathbf{H}^{(t)} \in$ $\mathbb{C}^{K \times K}$ denotes the channel frequency response (CFR) matrix corresponding to the $t$-th OFDM symbol, which can be represented as

$$
\mathbf{H}^{(t)}=\mathbf{F G}^{(t)} \mathbf{F}^{H}, \quad t=1, \cdots, T,
$$

where $\mathbf{F} \in \mathbb{C}^{K \times K}$ is the $K$-point DFT matrix and $\mathbf{G}^{(t)} \in$ $\mathbb{C}^{K \times K}$ is the channel impulse response (CIR) matrix of the $t$-th OFDM symbol and given by

$$
\mathbf{G}^{(t)}=\left[\begin{array}{cccccc}
g_{1,1}^{(t)} & 0 & \cdots & g_{1, N_{L}}^{(t)} & \cdots & g_{1,2}^{(t)} \\
g_{2,2}^{(t)} & g_{2,1}^{(t)} & 0 & \cdots & \cdots & g_{2,3}^{(t)} \\
\vdots & \ddots & \ddots & \ddots & \ddots & \vdots \\
0 & \cdots & 0 & g_{K, N_{L}}^{(t)} & \cdots & g_{K, 1}^{(t)}
\end{array}\right],
$$

where $g_{i, j}^{(t)}$ denotes the CIR of the $j$-th channel tap at time instant $i$.

Note that the diagonal elements of $\mathbf{H}^{(t)}$ are the desired frequency-domain channel responses, while the non-diagonal elements all represent ICI coefficients. Accordingly, we rewrite (1) into an element-wise form as

$$
\begin{array}{r}
Y_{k, t}=H_{k, k}^{(t)} X_{k, t}+\sum_{m=0, m \neq k}^{K-1} H_{k, m}^{(t)} X_{m, t}+W_{k, t}, \\
k=1, \cdots, K, \quad t=1, \cdots, T,
\end{array}
$$

where $Y_{k, t}$ is the $k$-th entry of $\mathbf{Y}_{t}, X_{k, t}$ is the $k$-th entry of $\mathbf{X}_{t}$, $W_{k, t}$ is the $k$-th entry of $\mathbf{W}_{t}$, and $H_{k, m}^{(t)}$ is the $(k, m)$-th entry of $\mathbf{H}^{(t)}$. The three terms of (4) represent the useful signal, the ICI, and the noise at the $k$-th subcarrier, respectively. An example of the CFR matrix $\mathbf{H}^{(t)}$ is shown in Fig. 1, where $f_{d}$ denotes the maximum normalized Doppler shift. It can be seen from Fig. 1 that the ICI power is mainly concentrated within a few adjacent subcarriers. In addition, there are also some non-ignorable entries in the bottom-left corner and the top-right corner, which is due to the cyclicity of the CP.

In this work, we adopt an efficient grid pilot pattern for channel estimation, i.e., the pilots occupy $K_{p}$ evenly spaced subcarriers of $T_{p}$ nonconsecutive OFDM symbols in each subframe. Note that this kind of pilot pattern has been commonly used in OFDM based wireless communication systems.

\section{Proposed Channel Estimation Network}

In this section, we develop a novel neural network based channel estimation method for the above introduced OFDM system. As depicted in Fig. 22 the proposed channel estimation method consists of three stages. For the first stage, we obtain initial channel estimates by performing the conventional LS estimation at the pilot symbols and linearly interpolating

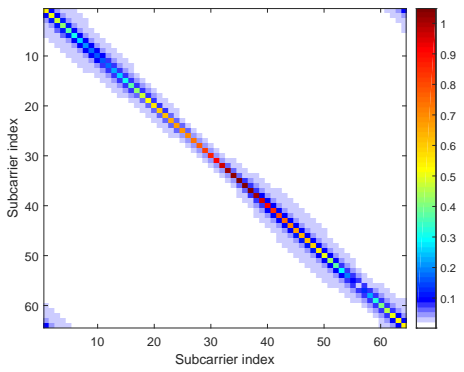

Fig. 1. Illustration of the normalized magnitude of a CFR matrix ( $K=$ $\left.64, f_{d}=0.1\right)$.

channels at the data symbols. For the second stage, we apply a preprocessing subnetwork PreDNN to incorporate the effect of ICI caused by the rapid channel variation. Finally, a residual learning based subnetwork CasResNet is cascaded with PreDNN to further improve the accuracy of the channel estimates. The details of the proposed two subnetworks and the corresponding training strategies are provided in the following.

\section{A. PreDNN}

It has been shown in (4) that the received signal at each subcarrier is subject to ICI. However, to the best of our knowledge, the effect of ICI is not considered in existing deep learning based fast time-varying channel estimation schemes, which can lead to performance degradation. Inspired by this fact, we propose a novel subnetwork PreDNN, which takes into account the non-negligible ICI and thus can remarkably enhance the channel estimation performance.

Specifically, PreDNN is a fully-connected neural network that involves only one hidden layer, which contains 32 neurons and adopts the rectified linear unit (ReLU) as the activation function. In order to incorporate the ICI into the network design, we embrace the information of both the subcarrier of interest and its adjacent subcarriers into the input of PreDNN. Since the ICI power is significant within only a few adjacent subcarriers, we can greatly simplify the input of PreDNN. Concretely, we first perform single-tap equalization for each subcarrier and obtain the estimate of $X_{k, t}$ as

$$
\hat{X}_{k, t}=\underset{X \in \mathcal{A}}{\arg \min }\left|\frac{Y_{k, t}}{\hat{H}_{k, t}}-X\right|^{2},
$$

where $\mathcal{A}$ is the modulation alphabet and $\hat{H}_{k, t}$ is the initial channel estimate of $H_{k, k}^{(t)}$ obtained in the first stage. For the $k$ th subcarrier of the $t$-th OFDM symbol, let us assume that the ICI mainly comes from $2 N_{I C I}$ neighbouring subcarriers, i.e., subcarrier $k-N_{I C I}$ to $k-1$ and subcarrier $k+1$ to $k+N_{I C I}$. Then, the received signals of all the $2 N_{I C I}+1$ subcarriers $Y_{k-N_{I C I}, t}, \cdots, Y_{k, t}, \cdots, Y_{k+N_{I C I}, t}$, the corresponding preestimated data symbols $\hat{X}_{k-N_{I C I}, t}, \cdots, \hat{X}_{k, t}, \cdots, \hat{X}_{k+N_{I C I}, t}$, and the initial channel estimate of the current subcarrier $\hat{H}_{k, t}$, are merged into a column vector as the input of PreDNN. The cyclicity in the frequency domain as shown in Fig. 1 is exploited for the first and last few subcarriers. For example, when $K=64$ and $N_{I C I}=1$, the input for the first 


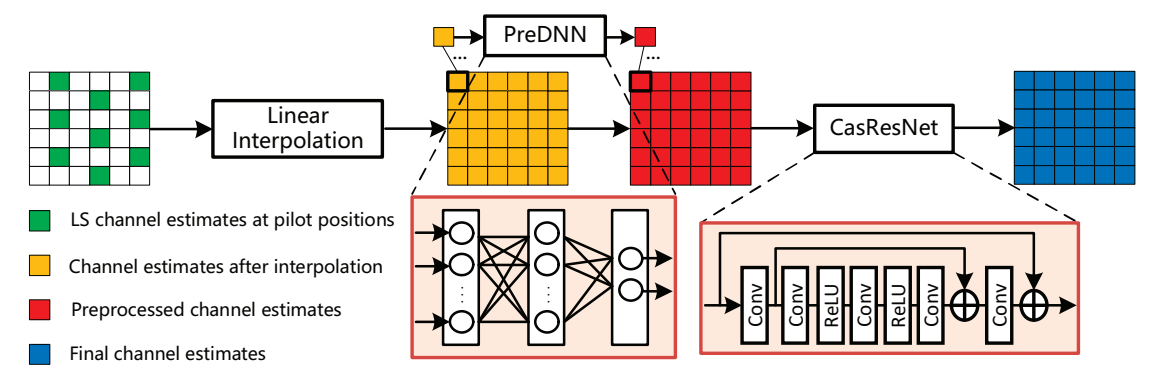

Fig. 2. Block diagram of the proposed channel estimation method.

subcarrier would be $\left[Y_{64, t}, Y_{1, t}, Y_{2, t}, \hat{X}_{64, t}, \hat{X}_{1, t}, \hat{X}_{2, t}, \hat{H}_{1, t}\right]^{T}$. Note that each complex element should be divided into real and imaginary parts to facilitate the use of software libraries such as Tensorflow. Hence, the input layer contains $8 N_{I C I}+6$ neurons in total. Likewise, the output layer contains 2 neurons, corresponding to the real and imaginary parts of the improved channel estimate $\tilde{H}_{k, t}$.

We note that the proposed PreDNN is used for improving the channel estimate achieved in the first stage in a subcarrierwise manner. In other words, once the network parameters are trained, they are shared by all subcarriers. Therefore, the scale of the network can be efficiently reduced and independent of the subframe size. Clearly, this can also be achieved by using a $1 \times 1$ convolutional layer.

\section{B. CasResNet}

After the preprocessing by PreDNN, the improved channel estimates of all the subcarriers are concatenated accordingly to form the 2D time-frequency channel response matrix $\tilde{\mathbf{H}} \in$ $\mathbb{C}^{K \times T}$. Regarding $\tilde{\mathbf{H}}$ as a noisy low-resolution image (equivalently transformed into $\mathbb{R}^{K \times T \times 2}$ ), we introduce CasResNet to enhance the resolution and alleviate the effect of noise.

CasResNet is a small-scale neural network based on the residual learning, which was first studied in [12] to address the vanishing gradient problem and improve the learning performance. The first layer is a convolutional layer using 8 filters of size $5 \times 5 \times 2$, which is expected to extract some low-level features from the original image $\tilde{\mathbf{H}}$, while more layers are stacked subsequently to further capture high-level features. Each of the following 3 convolutional layers has 8 filters of size $3 \times 3 \times 8$, with one ReLU layer inserted between each two of them. To be consistent with the input $\tilde{\mathbf{H}}$, the last convolutional layer uses 2 filters of size $5 \times 5 \times 8$ to generate a result of size $K \times T \times 2$. Specially, a double-residual-mapping nested architecture is built with two shortcut connections, by adding up the outputs of two certain layers. In the end, the output of CasResNet is reorganized according to the real and imaginary parts to get the final channel estimates $\breve{\mathbf{H}} \in \mathbb{C}^{K \times T}$.

Compared to the most relevant work ReEsNet [10], the superiority of the proposed CasResNet mainly lies in the following aspects:

1) The transposed convolution layer in ReEsNet for upsampling should be redesigned for a mismatched pilot pattern (including the number of pilots) or subframe size. Different from ReEsNet, we adopt the linear interpolation to implement pre-upsampling, which exploits the knowledge of traditional OFDM communications. Hence, CasResNet is model-driven and thus unaffected by the pilot pattern.

2) The parameter sharing property of PreDNN and the convolutional layers in CasResNet also enable our network to adapt to various subframe sizes without the need of redesigning, which guarantees the good compatibility.

3) By fully taking into account the impact of ICI, the preprocessing subnetwork PreDNN provides refined channel estimates for the cascaded subnetwork CasResNet. In this way, the network size of CasResNet can be reduced without compromising much performance, which thus efficiently lowers the complexity.

\section{Training Strategy}

Denote the function of PreDNN and CasResNet as $\mathcal{F}_{\text {Pre }}(\cdot)$ and $\mathcal{F}_{\text {Cas }}(\cdot)$, respectively. Then, the output of the proposed network can be expressed as

$$
\breve{\mathbf{H}}=\mathcal{F}_{\text {Cas }}\left(\mathcal{F}_{\text {Pre }}\left(\mathbf{Y}, \hat{\mathbf{X}}, \hat{\mathbf{H}}, \boldsymbol{\Theta}_{\text {Pre }}\right), \boldsymbol{\Theta}_{\text {Cas }}\right),
$$

where $\mathbf{Y}, \hat{\mathbf{X}}$ and $\hat{\mathbf{H}}$ denotes the received signals, the preestimated symbols and the initial channel estimates over the 2D time-frequency channel grid. In addition, $\Theta_{\text {Pre }}$ and $\boldsymbol{\Theta}_{\text {Cas }}$ are the trainable parameters of PreDNN and CasResNet, respectively. Considering that the subnetworks PreDNN and CasResNet are designed with distinct goals as introduced in Section III, we train them in a sequential manner instead of an end-to-end manner. we train the two subnetworks in a sequential manner. First, we train the parameters $\boldsymbol{\Theta}_{\text {Pre }}$ of PreDNN by minimizing the mean square error (MSE) between the estimated and true channel responses. The corresponding loss function is given by

$$
L\left(\boldsymbol{\Theta}_{\text {Pre }}\right)=\frac{1}{|\mathcal{S}|} \sum_{\overline{\mathbf{H}} \in \mathcal{S}}\|\tilde{\mathbf{H}}-\overline{\mathbf{H}}\|_{F}^{2},
$$

where $\mathcal{S}$ is the set of training samples, $\tilde{\mathbf{H}}$ and $\overline{\mathbf{H}}$ are the channel estimates refined by PreDNN and the true channel responses, respectively, and $\|\cdot\|_{F}^{2}$ denotes the Frobenius norm of a matrix. Then, with $\Theta_{\text {Pre }}$ fixed, we train CasResNet using the loss function defined as

$$
L\left(\boldsymbol{\Theta}_{\mathrm{Cas}}\right)=\frac{1}{|\mathcal{S}|} \sum_{\overline{\mathbf{H}} \in \mathcal{S}}\|\breve{\mathbf{H}}-\overline{\mathbf{H}}\|_{F}^{2},
$$


TABLE I

SYSTEM CONFIGURATION

\begin{tabular}{cc}
\hline Parameters & Values \\
\hline Carrier frequency & $2 \mathrm{GHz}$ \\
\hline Subcarrier spacing & $15 \mathrm{KHz}$ \\
\hline Number of subcarriers & 128 \\
\hline Number of OFDM symbols per subframe & 14 \\
\hline
\end{tabular}

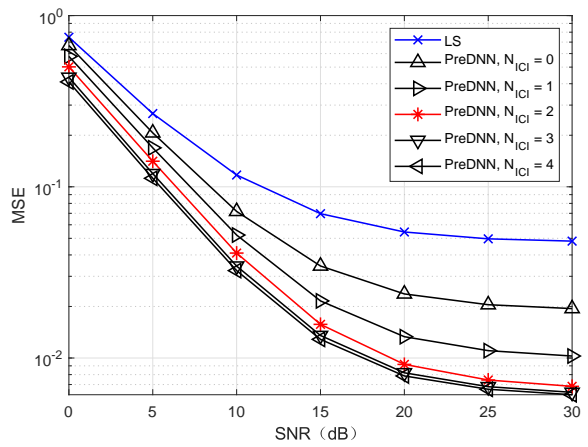

Fig. 3. MSE performance of PreDNN in terms of different $N_{I C I}$ 's with 84 pilots $\left(K_{p}=21, T_{p}=4\right)$.

where $\breve{\mathbf{H}}$ is the final channel estimates. Both subnetworks are trained by the well-established Adam optimizer, where the batch size, the initial learning rate, and the number of epochs are set to 200, 0.001, and 100, respectively.

\section{Simulation RESUlts}

We consider an OFDM system configured as in Table [] Quadrature phase-shift keying (QPSK) is used for modulation. For the training dataset, 12000 subframes are generated under the SNR of $10 \mathrm{~dB}$ with 10000 subframes for training and 2000 subframes for validation, each of which corresponds to a specific channel realization. The linear attenuation (LA) delay profile [13] with the Jakes Doppler spectrum is employed as the training channel model. In order to guarantee the generalization of the proposed network, the numbers of channel taps and the maximum Doppler shifts are randomly selected from 3 to 9 and 800 to $1200 \mathrm{~Hz}$, respectively. On the other hand, to demonstrate the robustness, the performance of the trained network is evaluated with 2000 testing subframes under a wide range of SNRs and a mismatched scenario, where the Extended Vehicle A (EVA) delay profile with 6 paths and the Jakes Doppler spectrum is adopted, and the maximum Doppler shift is set to $926 \mathrm{~Hz}$, i.e., a vehicle speed of $500 \mathrm{~km} / \mathrm{h}$.

\section{A. Performance Evaluation}

The refinement on the channel estimation by PreDNN can be partially attributed to the side information of the adjacent subcarriers. Hence, we first need to determine the number of adjacent subcarriers that should be taken into account. Accordingly, we present the MSE performance of PreDNN versus different $N_{I C I}$ 's in Fig. 3 It can be observed that a noticeable performance gain can be achieved as $N_{I C I}$ increases from 0 to 2 . After that, the performance of PreDNN gradually

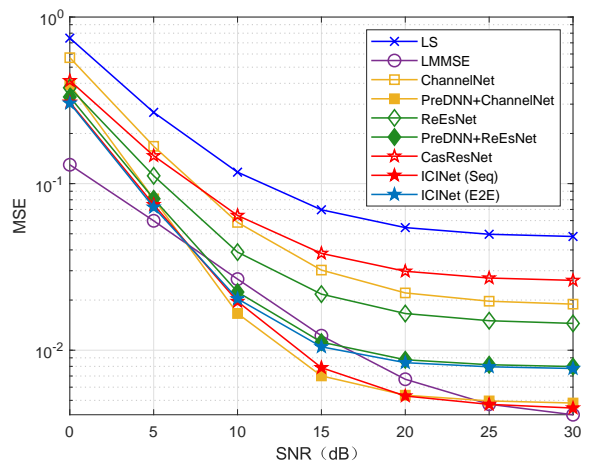

Fig. 4. MSE performance of different channel estimation methods with 84 pilots $\left(K_{p}=21, T_{p}=4\right)$ and $N_{I C I}=2$.

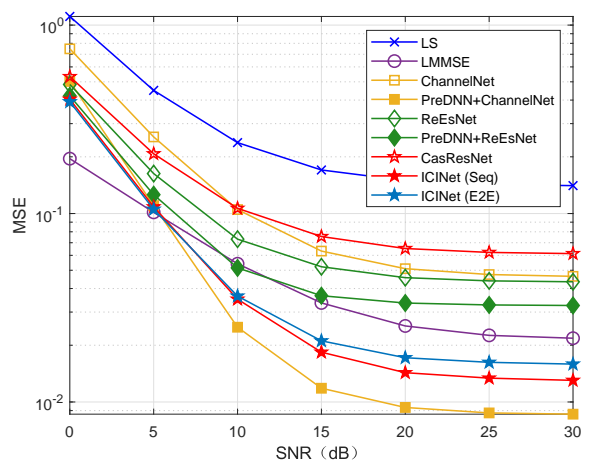

Fig. 5. MSE performance of different channel estimation methods with 48 pilots $\left(K_{p}=16, T_{p}=3\right)$ and $N_{I C I}=2$.

converges. Considering that a larger $N_{I C I}$ corresponds to a larger network and thus leads to higher complexity, we set $N_{I C I}=2$ during the simulation 1 It can also be found that even when $N_{I C I}=0$, PreDNN can still outperform the LS estimation, which indicates the powerful capability of deep neural networks.

The MSE performance of different channel estimation methods is compared in Figs. 4 and 5. For the case of 84 pilots, the LS estimation suffers from a high error floor due to the rapid channel variation, whereas the deep methods can achieve much better MSE performance. In particular, our proposed network ICINet, i.e., 'PreDNN+CasResNet', yields a much lower MSE than ChannelNet [9] and ReEsNet [10], which is also comparable to the linear minimum mean square error (LMMSE) estimation that requires the prior knowledge of channel statistics and noise variance 2 On the other hand, it can

\footnotetext{
${ }^{1}$ The value of $N_{I C I}$ mainly depends on the maximum Doppler shift, or, equivalently, the vehicle speed with given carrier frequency. In general, the higher the vehicle speed is, the more severe the ICI is, and the larger $N_{I C I}$ should be set. In practice, we can divide the range of all supported vehicle speeds into a few intervals and then establish a look-up table which maps the speed interval to the value of $N_{I C I}$ via offline simulations. Then, during the online channel estimation, we can first estimate the vehicle speed and then determine the value of $N_{I C I}$ using this table.

${ }^{2}$ The performance of the optimal MMSE estimate $\mathbb{E}(\overline{\mathbf{H}} \mid \mathbf{Y})$ is not provided considering the difficulty in obtaining an analytical form of the complicated posterior probability density function (PDF) $p(\overline{\mathbf{H}} \mid \mathbf{Y})$ of the general doubly selective OFDM channel. Moreover, it is also intractable to calculate the MMSE estimate numerically due to the large size of the OFDM channel.
} 


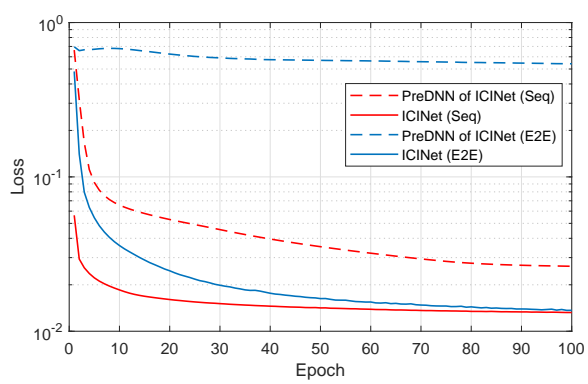

Fig. 6. Training loss comparison between the two training strategies with 84 pilots $\left(K_{p}=21, T_{p}=4\right)$ and $N_{I C I}=2$.

be seen that only using CasResNet leads to poor performance due to the limited network size, which validates the benefits from the preprocessing subnetwork PreDNN. Furthermore, we test the combination of PreDNN and some existing networks, i.e., 'PreDNN+ChannelNet' and 'PreDNN+ReEsNet', both of which exhibit better performance than the original networks. However, the additional gain obtained by cascading PreDNN with ReEsNet is less obvious, since PreDNN can only be applied at the pilots, resulting in limited improvement. When the number of pilots is reduced to 48 , the gaps between the deep learning based methods and the LS estimation become larger, and the proposed ICINet still performs considerably better than the existing networks and can even outperform the LMMSE estimation. Note that, for 'PreDNN+ChannelNet', the introduction of PreDNN makes it more attractive in terms of performance, but its complexity is also much higher as presented in the following subsection.

In addition, we can find that the sequentially trained ICINet, denoted by 'ICINet (Seq)', shows a better generalization than the end-to-end counterpart 'ICINet (E2E)' in the high SNR region, which indicates that the end-to-end training suffers overfitting due to the large number of network parameters to be optimized simultaneously. For further illustration, we compare the training loss of different training strategies in Fig. 6. Although the end-to-end training can reach almost the same loss as the sequential training, it suffers a slower convergence rate. This is due to the fact that, in the sequential training procedure, we have specified the training goal for the first subnetwork PreDNN, which can provide a good initial value for the cascaded subnetwork CasResNet and therefore facilitate its training. On the other hand, the endto-end training regards the whole network as a black box whose training performance highly relies on the training data. In fact, it can be observed that the PreDNN under the end-toend training does not even help improve the accuracy of the initial channel estimate.

\section{B. Complexity Analysis}

The complexity of different networks is compared in terms of the numbers of multiply-accumulate (MAC) operations and network parameters, which characterize the computational complexity and the memory usage, respectively. The results listed in Table $\Pi$ show that ICINet not only requires the fewest MAC operations but also has the fewest network parameters,
TABLE II

COMPLEXITY OF DifFERENT CHANNEl ESTIMATION NETWORKS

\begin{tabular}{ccc}
\hline Network & MAC Operations & Network Parameters \\
\hline ChannelNet & $2.41 \mathrm{e} 9$ & 676354 \\
\hline PreDNN+ChannelNet & $2.41 \mathrm{e} 9$ & 677156 \\
\hline ReEsNet & $8.15 \mathrm{e} 6$ & 44674 \\
\hline PreDNN+ReEsNet & $9.53 \mathrm{e} 6$ & 45476 \\
\hline CasResNet & $4.53 \mathrm{e} 6$ & 2562 \\
\hline ICINet & $\mathbf{5 . 9 1 e 6}$ & $\mathbf{3 3 6 4}$ \\
\hline
\end{tabular}

with advantages of up to several orders of magnitude over ChannelNet. Besides, it can be found that the combination with PreDNN does not bring much burden to the original networks.

\section{CONClusion}

In this letter, we have developed a novel OFDM channel estimation network for rapidly time-varying channels by incorporating the ICI into the network design. The proposed network ICINet consists of a preprocessing subnetwork PreDNN cascaded with a residual learning based subnetwork CasResNet, which can remarkably improve the accuracy of existing neural network based channel estimation schemes while with reduced computational complexity and memory usage. Moreover, the proposed network also exhibits good compatibility and robustness.

\section{REFERENCES}

[1] M. S. et al., "5G: A tutorial overview of standards, trials, challenges, deployment, and practice," IEEE J. Sel. Areas Commun., vol. 35, no. 6, pp. 1201-1221, Jun. 2017.

[2] T. O'Shea and J. Hoydis, "An introduction to deep learning for the physical layer," IEEE Trans. Cogn. Commun. Netw., vol. 3, no. 4, pp. 563-575, Dec. 2017.

[3] Z. Qin, H. Ye, G. Y. Li, and B. F. Juang, "Deep learning in physical layer communications," IEEE Wireless Commun., vol. 26, no. 2, pp. 93-99, Apr. 2019.

[4] H. He, S. Jin, C. Wen, F. Gao, G. Y. Li, and Z. Xu, "Model-driven deep learning for physical layer communications," IEEE Wireless Commun., vol. 26, no. 5, pp. 77-83, Oct 2019.

[5] H. Ye, G. Y. Li, and B. Juang, "Power of deep learning for channel estimation and signal detection in OFDM systems," IEEE Wireless Commun. Lett., vol. 7, no. 1, pp. 114-117, Feb. 2018.

[6] X. Gao, S. Jin, C. Wen, and G. Y. Li, "ComNet: Combination of deep learning and expert knowledge in OFDM receivers," IEEE Commun. Lett., vol. 22, no. 12, pp. 2627-2630, Dec. 2018.

[7] Y. Liao, Y. Hua, X. Dai, H. Yao, and X. Yang, "ChanEstNet: A deep learning based channel estimation for high-speed scenarios," in Proc. IEEE Int. Conf. Commun. (ICC), Shanghai, China, May 2019, pp. 1-6.

[8] Y. Liao, Y. Hua, and Y. Cai, "Deep learning based channel estimation algorithm for fast time-varying MIMO-OFDM systems," IEEE Commun. Lett., vol. 24, no. 3, pp. 572-576, Mar. 2020.

[9] M. Soltani, V. Pourahmadi, A. Mirzaei, and H. Sheikhzadeh, "Deep learning-based channel estimation," IEEE Commun. Lett., vol. 23, no. 4, pp. 652-655, Apr. 2019.

[10] L. Li, H. Chen, H. Chang, and L. Liu, "Deep residual learning meets OFDM channel estimation," IEEE Wireless Commun. Lett., vol. 9, no. 5 , pp. 615-618, May 2020.

[11] P. Schniter, "Low-complexity equalization of OFDM in doubly selective channels," IEEE Trans. Signal Process., vol. 52, no. 4, pp. 1002-1011, Mar. 2004.

[12] K. He, X. Zhang, S. Ren, and J. Sun, "Deep residual learning for image recognition," in Proc. IEEE Conf. Comput. Vis. Pattern Recognit. (CVPR), Las Vegas, NV, USA, Jun. 2016, pp. 770-778.

[13] X. Liu, H. Chen, S. Chen, and W. Meng, "Symbol cyclic-shift equalization algorithm - a CP-free OFDM/OFDMA system design," IEEE Trans. Veh. Technol., vol. 66, no. 1, pp. 282-294, Jan. 2017. 